\title{
Fundamentos para el aprendizaje significativo de la biodiversidad basados en el constructivismo y las metodologías activas
}

\section{Fundamentals for meaningful learning of biodiversity based on constructivism and active methodologies}

\author{
Ibone Santos-Ellakuria ${ }^{1}$
}

Fecha de recepción: 06/03/2019; Fecha de revisión: 05/07/2019; Fecha de aceptación: 10/07/2019

Cómo citar este artículo:

Santos-Ellakuria, I. (2019). Fundamentos para el aprendizaje significativo de la biodiversidad basados en el constructivismo y las metodologías activas. Revista de Innovación y Buenas Prácticas Docentes, 8(2), 90101.

Autor de Correspondencia: ibone.santos@ehu.eus

\section{Resumen:}

Existen evidencias de un escaso interés hacia las ciencias en el alumnado de la educación obligatoria. Asimismo, está descendiendo la frecuencia del contacto directo con la naturaleza en la niñez y en la adolescencia y el peso de la educación ambiental en el currículo es escaso. Ante estas tendencias, la presente investigación analiza cómo mejorar en la educación secundaria el proceso de enseñanzaaprendizaje de la biodiversidad. Se propone adoptar un enfoque constructivista y aplicar experiencias de aprendizaje basado en problemas, incluyendo prácticas de campo. De esta forma, se pretende aumentar la implicación del alumnado y su aprendizaje significativo, además de crear sinergias con otras materias para la comprensión integral de los aspectos socio-ambientales que regulan la dinámica del entorno.

Palabras clave: Biodiversidad, Constructivismo, ABP, Práctica de Campo

\section{Abstract:}

There is evidence of a little interest in science in students of compulsory education. Moreover, the frequency of direct contact with nature in childhood and adolescence is decreasing, and the relevance of environmental education in the curriculum is little. Faced with these trends, this research analyzes how to improve in secondary education the teaching-learning process of biodiversity. It is proposed to adopt a constructivist approach and apply problem-based learning experiences, including field practices outside the classroom. Thereby, it is intended to increase the involvement of students and their significant learning, in addition to creating synergies with other subjects for the comprehensive understanding of the socio-environmental aspects that regulates the dynamics of the environment.

Key Words: Biodiversity, Constructivism, PBL, Outdoor Education

\footnotetext{
1 Universidad del País Vasco (UPV/EHU) (España), ibone.santos@ehu.eus; CÓDIGO ORCID: https://orcid.org/0000-0002-4672-3373
} 


\section{INTRODUCCIÓN}

La biodiversidad, concepto que comprende la variabilidad de organismos vivos, así como la diversidad de procesos, interacciones y funciones que regulan su dinámica, proporciona a los seres humanos bienes y servicios esenciales para su subsistencia y bienestar (Díaz et al., 2006; Cardinale et al. 2012). Los recursos biológicos constituyen la base para gran parte de las actividades económicas, por ello, tanto la cantidad como la calidad de la biodiversidad son importantes a la hora de considerar el capital natural y los beneficios que éste proporciona. Es necesario desarrollar un adecuado nivel de conocimiento acerca del valor y las funciones de la biodiversidad en sus distintos niveles (genes, especies, ecosistemas, unidades de paisaje) para favorecer su conservación (Bermúdez \& Lindemann-Matthies, 2018). El reto de la conservación de la biodiversidad es de extrema complejidad, ya que durante las últimas décadas las actividades humanas para responder a la demanda creciente de alimento, agua dulce, materiales y energía han transformado los ecosistemas más rápida y extensamente que en ninguna otra época comparable de la historia humana (Díaz et al., 2006; Cardinale et al., 2012).

El Convenio sobre la Diversidad Biológica (Naciones Unidas, 1992) es el marco de referencia internacional para hacer frente a la pérdida de la biodiversidad. En las últimas décadas la sociedad ha ido concienciándose progresivamente de la importancia de poner freno al deterioro del patrimonio natural, pero una de las barreras a las que se enfrenta la puesta en valor de dicho patrimonio es la falta de conocimiento sobre los procesos que regulan la biodiversidad y sobre las causas y consecuencias de su disminución (Bermúdez \& Lindemann-Matthies, 2018). En este sentido, la educación es una herramienta imprescindible para adquirir valores como el respeto hacia los seres vivos y el medio ambiente (Vega-Marcote \& Álvarez-Suárez, 2009; Sousa et al., 2016; Casas-Jericó \& Puig i Baguer, 2017; Beery \& Jørgensen, 2018). La necesaria relación entre educación y conservación quedaba recogida en dicho Convenio, que concibe la educación como un elemento esencial para el mantenimiento y uso sostenible de la biodiversidad (Naciones Unidas, 1992).

El tratamiento de la biodiversidad en el entorno educativo ofrece potencialmente un amplio abanico de oportunidades tanto desde el punto de vista metodológico como de recurso educativo, ya que posibilita la aplicación de técnicas experimentales que conectan los conocimientos y destrezas de los alumnos con contextos de la vida real (Dopico \& García-Vázquez, 2011; Sousa et al., 2016; Jose et al., 2017; Pereira-García \& Muñoz-Lucas, 2017; Beery \& Jørgensen, 2018).

En la presente investigación realizamos un análisis de aquellos fundamentos didácticos que pueden impulsar con eficacia un aprendizaje significativo en materia de biodiversidad en la educación secundaria, etapa en la que el alumnado desarrolla en mayor medida la capacidad de comprender los elementos y procesos implicados en las ciencias naturales, así como un pensamiento crítico y reflexivo al respecto (TaccaHuamán, 2011). Proponemos la adopción de un enfoque constructivista y la aplicación de metodologías activas. Centramos nuestra atención en las posibilidades que ofrece la aplicación de experiencias de aprendizaje basado en problemas (ABP) que requieran a su vez de la realización de prácticas de campo. 


\section{EL TRATAMIENTO DE LA BIODIVERSIDAD EN EL ENTORNO EDUCATIVO}

En los últimos años la relevancia de la educación en materia de medio ambiente dentro del currículo de la enseñanza primaria y secundaria ha descendido en España (Benayas del Álamo et al., 2017) y existen reivindicaciones para aumentar la presencia de la educación ambiental en la futura ley educativa estatal (Rodríguez-Sánchez y RuizRobles, 2018). En cualquier caso, el sistema educativo español vigente contempla como uno de los objetivos de la enseñanza secundaria obligatoria que el alumnado adquiera valores y hábitos sociales que propicien el cuidado de los seres vivos y el medio ambiente, y contribuyendo a su conservación y mejora. En este sentido, y así queda reflejado en el currículo educativo vigente, es importante que los alumnos aprendan el significado del concepto de la biodiversidad en la materia de Biología y Geología y que adquieran una serie de valores hacia el respeto de la misma, comenzando por su entorno más cercano y cotidiano.

Por otro lado, se ha constatado, en paralelo al aumento del uso de las tecnologías digitales, un descenso del tiempo que pasan en contacto con la naturaleza los niños y adolescentes en países occidentales (Freire, 2001; Dillon et al., 2006; Larson et al., 2019). En cambio, existe un amplio consenso sobre los beneficios de realizar procesos de enseñanza-aprendizaje al aire libre, debido a efectos positivos en el desarrollo formativo del alumnado en las distintas etapas educativas (Dillon et al., 2006; Romero-Ariza, 2010; Freire, 2011; Frumklin et al., 2017; Seo/Birdlife, 2017; Beery y Jørgensen, 2018; Largo-Wight et al., 2018).

Asimismo, distintos autores han detectado un escaso de interés del estudiantado por las disciplinas científicas (Osborne et al., 2003; Gil-Pérez et al., 2005; Pozo-Municio \& Gómez-Crespo, 2006; Solbes et al., 2006; Rocard et al., 2007; Vázquez-Alonso y Manassero-Mas, 2011; Gil-Flores, 2012). La sobrecarga de programas, la enseñanza de conceptos abstractos sin experimentación, o la falta de conexión con las situaciones reales son apuntadas como algunas de las causas (Dillon et al., 2006: Rocard et al., 2007; Gavidia-Catalán, 2008; Solbes et al., 2007; Sousa et al., 2016). Si bien la percepción del alumnado respecto a las asignaturas de ciencias es positiva inicialmente, generalmente el interés desciende durante el desarrollo de la formación (Osborne et al., 2003; Gavidia-Catalán, 2008; Robles et al., 2015).

En esta línea, se ha constatado también un escaso nivel de motivación del alumnado hacia el estudio de los ecosistemas y seres vivos. Uno de los factores que contribuyen a esa desmotivación es la falta de familiarización que los alumnos tienen con su entorno natural más próximo, dado que pasan la mayor parte del tiempo dentro del aula (Romero-Ariza, 2010; Dopico \& García-Vázquez, 2011; Freire, 2011; Beery \& Jørgensen, 2018). Asimismo, a menudo las metodologías y estrategias de aprendizaje que se utilizan tampoco son adecuadas para despertar el interés y motivación hacia esta temática (García-Gómez \& Martínez-Bernat, 2010; Sousa et al., 2016; AmorteguiCedeño et al., 2017). En el caso concreto de la biodiversidad, los problemas que presenta su aprendizaje en España son variados, como la falta de comprensión de los aspectos sociales y económicos vinculados a la pérdida de la biodiversidad, el tratamiento incompleto que realizan los libros de texto a nivel conceptual, o la falta de formación del profesorado (García-Gómez \& Martínez-Bernat, 2010; Fuentes-Silveira, 2015; Bermúdez et al., 2014, 2016; Amortegui-Cedeño et al., 2017; Manassero-Mas \& Vázquez-Alonso, 2017; García-Barros, 2018). 
Si bien las investigaciones sobre la biodiversidad y su problemática en el ámbito educativo están emergiendo desde tiempos recientes, distintos autores coinciden en la necesidad de dotar desde la educación científica al alumnado de unas competencias teórico-prácticas que les permita tener una actitud crítica y tomar decisiones al respecto (Gavidia-Catalán, 2008; García-Gómez \& Martínez-Bernat, 2010; Bermúdez et al., 2014, 2016; Manassero-Mas \& Vázquez-Alonso, 2017; Bermúdez \& Lindemann-Matthies, 2018). No obstante, algunos estudios afirman que los alumnos tienen escaso conocimiento sobre esta problemática ambiental, especialmente en el contexto local, y tienen dificultades a la hora de integrar los aspectos sociales y económicos consustanciales (Vega-Marcote \& Álvarez-Suárez, 2009; Fuentes-Silveira, 2015; Bermúdez et al., 2016; Jose et al., 2017).

Asimismo, la enseñanza de la biodiversidad debe atender no solo al significado del concepto y la identificación de sus componentes, sino también a los procesos que la regulan y la relación que se establece con el ser humano. Así se deberían estudiar los bienes y servicios que aporta la biodiversidad a la sociedad y la relación que guarda su conservación con la calidad de vida de las personas (Van Weelie \& Wals, 2002; Martínez-Bernat \& García-Gómez, 2009; Bermúdez et al., 2016). Para ello, han de promoverse propuestas educativas con metodología de enseñanza-aprendizaje concretas que promuevan el conocimiento del contexto natural local (Martínez-Bernat \& García-Gómez, 2009; Fuentes-Silveira, 2015).

\section{EL CONSTRUCTIVISMO Y LAS METODOLOGÍAS ACTIVAS}

Una propuesta de intervención educativa eficaz debe asentarse sobre unas bases psicopedagógicas sólidas y en ciencias el constructivismo constituye un modelo de referencia (Driver, 1988; Carretero, 1993; Furió-Mas et al., 1994; Díaz-Barriga \& Hernández-Rojas, 2002; Cruz-Guzmán, 2011; Serrano \& Pons, 2011). A través de este modelo, el alumno va adquiriendo los conocimientos en el contexto donde ocurre el aprendizaje y vinculado a las experiencias de las que participa (Driver, 1988; DíazBarriga \& Hernández-Rojas, 2002; Cruz-Guzmán, 2011; Serrano \& Pons, 2011). Así, en el aprendizaje constructivista, el alumno toma un papel activo y va construyendo su propio conocimiento mediante un conjunto de experiencias que le permiten desarrollar las destrezas e ideas deseadas (Driver, 1988; Díaz-Barriga \& Hernández-Rojas, 2002; Cruz-Guzmán, 2011; Serrano \& Pons, 2011). Dicha construcción de conocimiento se ve facilitada por las relaciones que el estudiante establece entre los nuevos conceptos y los que previamente había adquirido (Ausubel, 2002). Mientras, el profesor ejerce de guía en el aprendizaje y promueve la realización de actividades prácticas (Díaz-Barriga \& Hernández-Rojas, 2002).

Desde el enfoque del constructivismo social se parte de la base de que la interacción de los alumnos entre sí y con el entorno favorece el aprendizaje (Carretero, 1993). Esta corriente del constructivismo es interesante para la enseñanza de la Biología y Geología y, en concreto, para la biodiversidad, ya que contempla las dimensiones ética y social presentes también en los problemas ambientales. El tratamiento de la biodiversidad y su pérdida exigen unos planteamientos educativos integrales que permitan reflexionar al alumno sobre los aspectos ambivalentes característicos de las decisiones ambientales (García-Gómez \& Martínez-Bernat, 2010). 
Unidas al modelo constructivista, las metodologías activas son una manera adecuada de presentar los contenidos ya que entienden la enseñanza como algo constructivo donde el alumno forma parte activa como protagonista de su propio proceso de aprendizaje, mientras que el docente actúa de mediador y guía. Las metodologías activas permiten al alumnado ser el centro del proceso de enseñanza-aprendizaje y contextualizar los problemas del mundo real al cual se enfrentará en el futuro (Tribó, 2008; De Miguel, 2013; Dole et al., 2016). De esta manera, contribuyen a aumentar la motivación y participación del individuo, así como a mejorar su habilidad argumentativa, desarrollando con ello la competencia de aprender a aprender con sentido crítico sobre su actuación (Fernández-March, 2006; Manassero-Mas \& Vázquez-Alonso, 2017).

Además, esta metodología otorga importancia a la puesta en práctica de los conocimientos mediante la resolución de problemas reales y cercanos al estudiante. Asimismo, con estas metodologías el alumnado asimila y retiene los conceptos en mayor medida y se consiguen mejores resultados de motivación y participación, ya que se le da la oportunidad de contrastar opiniones y argumentar posturas con el resto de compañeros (Fernández-March, 2006; Abellán-Toledo \& Herrada-Valverde, 2016; Dole et al., 2016).

Así, las metodologías activas podrían ser útiles para revertir la actual tendencia del desinterés del alumnado hacia las ciencias (Pozo-Municio \& Gómez-Crespo, 2006). Distintos autores han señalado ciertas pautas a seguir por el profesorado de ciencias para aumentar el interés e implicación del alumnado, como contextualizar la materia y exponer sus utilidades en la vida real, impulsar la educación basada en la investigación para generar curiosidad, o facilitar espacios y prácticas para la experimentación dentro y fuera del centro escolar (Rocard et al., 2007; Gavidia-Catalán, 2008; Amórtegui et al., 2017).

\section{EL APRENDIZAJE BASADO EN PROBLEMAS Y LA PRÁCTICA DE CAMPO}

Partiendo de la premisa de que la construcción del conocimiento científico tiene por objeto la búsqueda de soluciones para comprender mejor la realidad, las estrategias didácticas de la corriente constructivista pueden diseñarse como situaciones-problema (Furió-Mas et al., 1994). Así, el aprendizaje basado en problemas (ABP) encuentra un fundamento en el constructivismo y resulta una alternativa válida para compensar los problemas que conlleva la enseñanza tradicional de la ciencia (Barrows, 1986; MoralesBueno \& Landa-Fitzgerald, 2004; Pantoja-Castro \& Covarrubias-Papahiu, 2013; Dole et al., 2016). 
El $A B P$ es un método didáctico basado en el principio de plantear problemas reales como punto de partida para la adquisición e integración de los nuevos conocimientos (Barrows, 1986). Sus características fundamentales son las siguientes: i) el alumno está en el centro del aprendizaje y es el responsable de identificar qué es lo que necesita aprender y cómo conseguir la información necesaria con el objetivo último de resolver el problema que se le plantea; ii) los alumnos trabajan en grupos pequeños, de 5 a 8 personas, en los que se reparten las tareas y todos asumen la responsabilidad y el compromiso de conseguir los objetivos previstos. Esto les permite adquirir experiencia en el trabajo en equipo; iii) los profesores actúan como guías del proceso de aprendizaje planteando a los alumnos preguntas que les hagan cuestionarse sobre cuál es la mejor manera de resolver el problema propuesto; iv) el problema planteado se resuelve recurriendo a la información adquirida en distintas asignaturas, por lo que el método sirve para integrar conocimientos de varias disciplinas; v) se plantean problemas muy cercanos a la vida real, de tal forma que el alumno pueda poner en práctica los conocimientos en un contexto auténtico donde se verá en un futuro; y vi) los estudiantes adquieren la nueva información de forma autodirigida con sus propias estrategias, conocimientos previos, estudio e investigación y con el profesor como facilitador del aprendizaje (Morales-Bueno \& Landa-Fitzgerald, 2004; García de la Vega, 2012; Pantoja-Castro \& Covarrubias-Papahiu, 2013; Pereira-García\&y Muñoz-Lucas, 2017).

Al aplicarse al aprendizaje de la biodiversidad, y dado que el ABP parte del planteamiento de un problema concreto, es oportuno que dicho ABP incluya la práctica de campo fuera del aula, para que así el alumnado experimente un contacto directo con la situación problema. En la educación reglada, el trabajo de campo es una labor pedagógica que implica la exploración del entorno cercano al centro educativo, aspecto muy relevante en la enseñanza de la Biología (Castro-Moreno \& Valbuena-Ussa, 2007). Existen múltiples evidencias de que el aprendizaje fuera del aula aumenta el valor educativo de las actividades, ya que ayuda a comprender mejor los conceptos explicados con metodología y recursos convencionales, y supera las barreras que imponen las limitaciones del aula (Romero-Ariza, 2010; García de la Vega, 2010; Dopico \& García-Váquez, 2011; Montero-Vilar et al. 2012; Amórtegui et al., 2017). También existen evidencias de que los problemas ambientales globales como la pérdida de biodiversidad, conviene tratarlos en contextos locales, ya que permiten aprender teniendo un contacto directo con el entorno inmediato (Pérez de Sánchez \& RodríguezPizzinato, 2006; Dopico \& García-Vázquez, 2011; Montero-Vilar et al., 2012). 
Por otro lado, existe constancia de que la práctica de campo es una estrategia didáctica útil, dado que acerca de manera consciente al alumnado a la realidad, permitiéndole así comprender con mayor facilidad los procesos, elementos e implicaciones de la biodiversidad en el entorno (Dopico \& García-Vázquez, 2011; Pérez de Sánchez \& Rodríguez-Pizzinato, 2006; Amórtegui-Cedeño et al., 2017; Jose et al., 2017;). Así, la biodiversidad preferentemente ha de abordarse en un contexto conocido por el alumno, de tal manera que éste pudiera establecer una relación práctica entre los problemas y sus implicaciones reales. De esta forma, se da un enfoque más práctico al tratamiento del tema, más cercano a la realidad y, por tanto, más motivador para el estudiante, lo cual puede mejorar la percepción que éste tiene respecto a la ciencia. Además, la educación al aire libre en contextos locales favorece la puesta en valor del patrimonio natural del lugar y genera sentimientos de apego ya que permite a los estudiantes poner en práctica sus conocimientos y buscar soluciones para problemas de situaciones reales. Así los alumnos van consolidando sus argumentos sobre la idoneidad de preservar el medio natural y se van formando como personas comprometidas con la conservación del medio ambiente, que entienden su funcionamiento y toman consciencia sobre su propia capacidad de participar en la toma de decisiones (Dillon et al., 2006; García-Gómez \& Martínez-Bernat, 2010; AmórteguiCedeño et al., 2017).

\section{CONCLUSIONES}

Por todo lo expuesto, consideramos de gran interés para el profesorado de educación secundaria el diseño y aplicación de al menos una experiencia ABP para la enseñanza de la biodiversidad, y que dentro de la misma, además de las características mencionadas anteriormente, se incluya una práctica de observación, búsqueda y toma de datos en el campo como una de las fuentes de información a manejar por el alumnado para la resolución de dicha ABP.

Es preciso también aprovechar la oportunidad de que, además de la biodiversidad, existen otros aspectos del medio que son susceptibles de ser comprendidos de manera proactiva por el alumnado de la educación secundaria mediante la combinación de ABP y prácticas de campo, siendo posible abarcar el conjunto de factores ambientales, socioeconómicos, históricos y culturales que confluyen en el espacio (Pérez de Sánchez \& Rodríguez-Pizzinato, 2006; García de la Vega, 2010, 2012; Casas-Jericó \& Erneta-Altarriba, 2016; Llancavil-Llancavil, 2018). Por ello, proponemos que se programen experiencias ABP y prácticas de campo en la misma área de estudio en las distintas asignaturas de la educación secundaria que contribuyen al conocimiento del medio, con el fin de generar sinergias positivas para un aprendizaje más transversal e integral del conjunto de las variables sociales y ambientales que influyen en las características y en la dinámica del entorno. Esto favorecería una mayor integración de conocimientos de varias disciplinas. Además, las prácticas de campo pueden ser aprovechadas para el uso y elaboración de cartografías, con mapas tanto en papel como en formato digital con tecnologías de información geográfica. Esto permite al alumnado aprender a situarse en el área de estudio y a obtener la ubicación de los datos de campo recopilados, favoreciendo el desarrollo de las competencias espaciales (De Miguel, 2013). 
De esta manera, proponemos que la combinación de ABP y práctica de campo es relevante para catalizar el aprendizaje significativo en materia de biodiversidad, así como para aumentar el interés del alumnado, ya que requiere de la interacción proactiva de éste con el medio natural y social que le rodea. Su diseño ha de atender, además de al significado del concepto de biodiversidad y a la identificación de sus componentes, a los procesos que la regulan y la relación que se establece con el ser humano. Esto se ve facilitado por el aprendizaje transversal en combinación con otras materias, mediante la combinación de ABP y prácticas de campo sobre distintos aspectos humanos y ambientales en la misma área de estudio. El área de estudio de las prácticas de campo idealmente debiera situarse cerca del centro escolar y estar compuesta por sectores con distinto grado de intervención humana (sectores naturales, rurales y urbanos), con el fin de integrar el papel de los aspectos ambientales, socioeconómicos, históricos y culturales que influyen en la dinámica de la biodiversidad.

Con todo ello se persigue que el alumnado pueda desarrollar conclusiones sobre los bienes y servicios que aporta la biodiversidad a la sociedad y sobre la relación que guarda su conservación con la calidad de vida de las personas. Este tipo de metodología docente es especialmente necesario en el contexto actual, dado que contribuye a aumentar la motivación del alumnado, su contacto con la naturaleza en el entorno local y su pensamiento crítico sobre los problemas ambientales. Se trata de aspectos fundamentales para el alumnado desarrolle valores y hábitos sociales que propicien la conservación y mejora de los seres vivos y el medio ambiente.

\section{REFERENCIAS}

Abellán-Toledo, Y. \& Herrada-Valverde, R. I. (2016). Innovación educativa y metodologías activas en educación secundaria: la perspectiva de los docentes de lengua castellana y literatura. Fuentes, 18, 65-76. Recuperado de: https://revistascientificas.us.es/index.php/fuentes/article/view/2371

Amortegui-Cedeño, E.F., Mayoral García-Berlanga, O. \& Gavidia-Catalán, V. (2017). Aportaciones de las Prácticas de Campo en la formación del profesorado de Biología: un problema de investigación y una revisión documental. Didáctica de las Ciencias Experimentales y Sociales, 32, 153-169. Recuperado de: http://dx.doi.org/10.7203/dces.32.9940

Arboleya-García, E. \& Dopico-Rodríguez, E. (2017). Superando las barreras físicas del aula: recursos naturales y TIC. Revista Iberoamericana de Educación, 75, 71-88. Recuperado de: https://rieoei.org/RIE/article/view/1356

Ausubel, D.P. (2002). Adquisición y retención del conocimiento. Una perspectiva cognitiva. Barcelona: Paidós.

Barrows, H.S. (1986). A taxonomy of problem-based learning methods. Medical Education, 20, 481-486. DOI: http://dx.doi.org/10.1111/j.13652923.1986.tb01386.x

Beery, T. \& Jørgensen, K.A. (2018). Children in nature: sensory engagement and the experience of biodiversity. Environmental Education Research, 24, 13-25. DOI: http://dx.doi.org/10.1080/13504622.2016.1250149

Benayas Del Álamo, J., Marcén-Albero, C., Alba-Hidalgo, D. \& Gutiérrez-Bastida, J.M. (2017). Educación para la sostenibilidad en España: Reflexiones y propuestas. Madrid: Fundación Alternativas. Recuperado de: https://www.fundacionalternativas.org/public/storage/opex_documentos_archivo s/81ef826c30f2322a5c9c8536a50faf20.pdf 
Bermúdez, G.M.A., De Longhi, A.L., Díaz, S. \& Gavidia-Catalán V. (2014) La transposición del concepto de diversidad biológica. Un estudio sobre los libros de texto de la educación secundaria española. Enseñanza de las Ciencias, 32, 285-302. DOI: http://dx.doi.org//10.5565/rev/ensciencias.1129

Bermúdez, G.M.A., De Longhi, A.L., Díaz, S. \& Gavidia-Catalán V. (2016). El tratamiento de los bienes y servicios que aporta la biodiversidad en manuales de la educación secundaria española: Un estudio epistemológico. Eureka sobre enseñanza y divulgación de las ciencias, 13, 527-543. Recuperado de: https://rodin.uca.es/xmlui/handle/10498/18495

Bermúdez, G.M.A. \& Lindemann-Matthies, P. (2018) "What matters is species richness"-High school students' understanding of the components of biodiversity. Research in Science Education. DOI: http://dx.doi.org//10.1007/s11165-018-9767-y

Carretero, M. (1993). Constructivismo y educación. Zaragoza: Edelvives.

Cardinale, B.J., Duffy, J.E., Gonzalez, A. et al. (2012). Biodiversity loss and its impact on humanity. Nature, 486, 59-67. DOI: http://dx.doi.org//10.1038/nature11148

Casas-Jericó, M. \& Erneta-Altarriba, L. (2016). El paisaje en la educación secundaria obligatoria. Una oportunidad educativa en el cambio curricular LOE-LOMCE. Didáctica Geográfica, 16, 45-71. Recuperado de: http://www.agegeografia.es/didacticageografica/index.php/didacticageografica/article/view/295

Casas-Jericó, M. \& Puig I Baguer, J. (2017). El impacto ambiental: Un despertar ético valioso para la educación. Teoría de la Educación, 29, 101-128. Recuperado de: http://revistas.usal.es/index.php/1130-3743/article/view/teoredu291101128

Castro-Moreno, J. \& Valbuena-Ussa, E. (2007). ¿Qué biología enseñar y comó hacerlo? Hacia una resignificación de la biología escolar. Tecné, Episteme y Didaxis, 22, 126-145. DOI: http://dx.doi.org/10.17227/ted.num22-385

Cruz-Guzmán, M. (2011). Diseño práctico de una Unidad Didáctica en el área de las Ciencias Experimentales enmarcado en un proceso de enseñanza-aprendizaje activo y constructivista. Campo Abierto, 30, 141-163. Recuperado de: http://hdl.handle.net/11441/38616

De Miguel, R. (2013). Aprendizaje por descubrimiento, enseñanza activa y geoinformación. Hacia una didáctica de la geografía innovadora. Didáctica Geográfica, 14, 17-36. Recuperado de: http://www.agegeografia.es/didacticageografica/index.php/didacticageografica/article/view/230

Díaz, S., Fargione, J., Chapin, F.S. \& Tilman, D. (2006). Biodiversity loss threatens human well-being. PLoS Biology, 4, e277. DOI: http://dx.doi.org/10.1371/journal.pbio.0040277

Díaz-Barriga, F. \& Hernández-Rojas, G. (2002). Estrategias docentes para un aprendizaje significativo: una interpretación constructivista. México D.F.: McGraw-Hill.

Dillon, J., Rickinson, M., Teamey, K., Choi, M. \& Benefield, P. (2006). The value of outdoor learning: evidence from research in the UK and elsewhere. School Science Review, 87, 107-111.

Dole, S., Bloom, L. \& Kowalske, K. (2016). Transforming pedagogy: Changing perspectives from teacher-centered to learner-centered. Interdisciplinary Journal of Problem-Based Learning, 10, 45-58. DOI: http://dx.doi.org/10.7771/1541 5015.1538

Dopico, E. \& García-Vázquez, E. (2011). Leaving the classroom: a didactic framework for education in environmental sciences. Cultural Studies of Science Education, 6, 311-326. DOI: http://dx.doi.org/10.1007/s11422-010-9271-9

Driver, R. (1988). Un enfoque constructivista para el desarrollo del currículo en ciencias. Enseñanza de las Ciencias, 6, 109-120. Recuperado de: https://www.raco.cat/index.php/Ensenanza/article/view/51075 
Fernández-March, A. (2006). Metodologías activas para la formación de competencias. Educatio Siglo XXI, 24, 35-56. Recuperado de: https://revistas.um.es/educatio/article/view/152

Freire, H. (2011). Educar en verde. Ideas para acercar a niños y niñas a la naturaleza. Barcelona: Graó.

Frumkin, H., Bratman, G.N., Breslow, S.Jo., Cochran, B., Kahn, P.H., Lawler, J.J., Levin, P.S., Tandon, P.S.; Varanasi, U.; Wolf, K.L. \& Wood, S.A. (2017). Nature contact and human health: A research agenda. Environmental Health Perspectives, 125, 075001. DOI: http://dx.doi.org/10.1289/EHP1663

Fuentes-Silveira, M. J. (2015). La diversidad en el marco de la didáctica de las ciencias naturales: una experiencia en el aula de educación secundaria obligatoria (Tesis Doctoral). A Coruña: Universidade da Coruña. Recuperado de: https://ruc.udc.es/dspace/handle/2183/14786

Fuentes-Silveira, M. J. \& García-Barros, S. (2009). El ser humano y la biodiversidad en el ecosistema: validación de una experiencia de aula. Enseñanza de las Ciencias, $n^{\circ}$ extra, 1496-1502. Recuperado de: https://www.raco.cat/index.php/Ensenanza/article/view/294036

Furió-Mas, C. J., Iturbe-Barrenetxea, J. \& Reyes-Martín, J. V. (1994). Contribución de la resolución de problemas como investigación al paradigma constructivista de aprendizaje de las ciencias. Investigación en la Escuela, 24, 89-99. Recuperado de: http://hdl.handle.net/11441/59624

García-Barros, S. (2018). La biodiversidad: una reflexión sobre el contenido que se debe enseñar. Alambique, 94, 7-12.

García-Carmona, A. \& Acevedo-Díaz, J.A. (2018). The nature of scientific practice and science education: Rationale of a set of essential pedagogical principles. Science \& Education, 27, 435-455. DOI: http://dx.doi.org/10.1007/s11191-018-9984-9

García de la Vega, A. (2010). Aplicación didáctica del aprendizaje basado en problemas al análisis geográfico. Didácticas Específicas, 2, 41-59. Recuperado de: https://revistas.uam.es/didacticasespecificas/article/view/9201/9472

García de la Vega, A. (2012). El aprendizaje basado en problemas en los itinerarios didácticos vinculados al patrimonio. Educación y Futuro, 27, 155-175. Recuperado de: http://hdl.handle.net/10486/661668

García-Gómez, J. \& Martínez-Bernat, F.J. (2010). Cómo y qué enseñar de la biodiversidad en la alfabetización científica. Enseñanza de las Ciencias, 28, 175184.

de: https://www.raco.cat/index.php/Ensenanza/article/view/199611

Gavidia-Catalán, V. (2008). Las actitudes en la educación científica. Didáctica de las Ciencias Experimentales y Sociales, 22, 53-66. Recuperado de: https://ojs.uv.es/index.php/dces/article/view/2420

Gil-Flores, J. (2012). Actitudes del alumnado español hacia las ciencias en la evaluación PISA 2006. Enseñanza de las Ciencias, 30, 131-152. Recuperado de: https://www.raco.cat/index.php/Ensenanza/article/view/254507

Gil-Pérez, D., Macedo, B., Martínez-Torregrosa, J., Sifredo, C., Valdés, P. \& Vilches, A. (Eds.). (2005). ¿Cómo promover el interés por la cultura científica? Una propuesta didáctica fundamentada para la educación científica de jóvenes de 15 a 18 años. Santiago de Chile: OREALC/UNESCO. Recuperado de: https://unesdoc.unesco.org/ark:/48223/pf0000139003

Jose, S., Patrick, P.G. \& Moseley, C. (2017). Experiential learning theory: The importance of outdoor classrooms in environmental education. International 
Journal of Science Education, 7, 269-284. DOI: http://dx.doi.org/10.1080/21548455.2016.1272144

Largo-Wight, E., Guardino, C., Wludyka, P.S., Hall, K.W., Wight, J.T. \& Merten, J.W. (2018). Nature contact at school: The impact of an outdoor classroom on children's well-being. International Journal of Environmental Health Research, 28, 653-666.

DOI: http://dx.doi.org///10.1080/09603123.2018.1502415

Larson, L.R., Szczytko, R., Bowers, E.P., Stephens, L.E., Stevenson, K.T. \& Floyd, M.F. (2019). Outdoor time, screen time, and connection to nature: Troubling trends among rural youth? Environment and Behavior: http://dx.doi.org/10.1177/0013916518806686

Llancavil-Llancavil, D. (2018). Trabajo de campo: Producción y significancia de conocimiento geográfico. Perspectiva Educacional, 57, 143-162. http://www.perspectivaeducacional.cl/index.php/peducacional/article/view/625

Martínez-Bernat, F.J. \& García-Gómez, J. (2009). Análisis del tratamiento didáctico de la biodiversidad en los libros de texto de Biología y Geología en Secundaria. Didáctica de las Ciencias Experimentales y Sociales, 23, 109-122. Recuperado de: https://ojs.uv.es/index.php/dces/article/view/2410

Manassero-Mas, M.A. \& Vázquez-Alonso, A. (2017). ¿Hay contenidos de naturaleza de la ciencia y la tecnología y pensamiento crítico en los currículos (españoles) actuales? Enseñanza de las Ciencias, $n^{\circ}$ extra, 509-514. Recuperado de: https://www.raco.cat/index.php/Ensenanza/article/view/334607

Montero-Vilar, S., Brown, D. \& García-Rodeja, I. (2012). Monte multifuncional: una actividad para trabajar la conservación de la naturaleza en contextos locales. Enseñanza de las Ciencias, $n^{\circ}$ extra, 2368-2374. Recuperado de: https://www.raco.cat/index.php/Ensenanza/article/view/307877

Morales-Bueno, P., y Landa-Fitzgerald, V. (2004). Aprendizaje basado en problemas. Theoria, 13, 145-157. https://www.redalyc.org/articulo.oa?id=29901314

Osborne, J., Simon, S. \& Collins, S. (2003). Attitudes towards science: A review of the literature and its implications. International Journal of Science Education, 25, 1049-1079. DOI: http://dx.doi.org//10.1080/0950069032000032199

Pantoja-Castro, J. \& Covarrubias-Papahiu, P. (2013). La enseñanza de la biología en el bachillerato a partir del aprendizaje basado en problemas (ABP). Perfiles Educativos, 139, 93-109. Recuperado de: http://www.scielo.org.mx/pdf/peredu/v35n139/v35n139a7.pdf

Pérez De Sánchez, A. \& Rodríguez-Pizzinato, L. (2006). La salida de campo: una manera de enseñar y aprender geografía. Geoenseñanza, 11, 229-234. Recuperado de: https://www.redalyc.org/articulo.oa?id=36012425008

Pereira-García, F.J. y Muñoz-Lucas, M.I. (2017). El Aprendizaje Basado en Problemas como herramienta para el estudio de los fenómenos geológicos. Infancia, Educación y Aprendizaje, 3, 492-498. DOI: http://dx.doi.org/10.22370/ieya.2017.3.2.769

Pozo-Municio, J. I. \& Gómez-Crespo, M. A. (2006). Aprender y enseñar ciencia: del conocimiento cotidiano al conocimiento científico. Madrid: Morata.

Robles, A., Solbes, J., Cantó, J.R. \& Lozano, O.R. (2015). Actitudes de los estudiantes hacia la ciencia escolar en el primer ciclo de la Enseñanza Secundaria Obligatoria. Electrónica de Enseñanza de las Ciencias, 14, 361-376. Recuperado de: http://reec.uvigo.es/volumenes/volumen14/REEC_14_3_6_ex939.pdf

Rodríguez-Sánchez, M. \& Ruiz-Robles, R. (Coords.) (2018). Retos de la educación para la sostenibilidad en el siglo XXI. Madrid: Conama. Recuperado de: http://www.conama.org/download/bancorecursos/documentos/GTEAC_RETOS \%20ES\%20EN\%20EL\%20SIGLO\%20XXI.PDF

Romero-Ariza, M. (2010). El aprendizaje experiencial y las nuevas demandas formativas de antropología experimental, 10, 89-102. Recuperado de: https://revistaselectronicas.ujaen.es/index.php/rae/article/view/1970/1718 
Rocard, M., Hemmo, V, Csermely, P., Jorde, D., Lenzen, D. \& Walwerg-Henriksson, H. (2007). Science education now: A renewed pedagogy for the future of Europe. Brussels: European Commission. Recuperado de: https://ec.europa.eu/research/science-society/document_library/pdf_06/reportrocard-on-science-education_en.pdf

SEO/Birdlife (2017). Pacto por la educación: Propuesta de Seo/BirdLife para educar por un desarrollo sostenible. Madrid: Sociedad Española de Ornitología. Recuperado de: https://naturalezaenlasaulas.seo.org/wp-content/uploads/2018/03/Pactoeducaci\%C3\%B3n-SEO_BLRed.pdf

Serrano, J. M. \& Pons, R. M. (2011). El constructivismo hoy: enfoques constructivistas en educación. Revista Electrónica de Investigación Educativa, 13, 1-27. Recuperado de: https://redie.uabc.mx/redie/article/view/268/708

Solbes, J., Montserrat, R. \& Furió-Mas, C. (2007). El desinterés del alumnado hacia el aprendizaje de la ciencia: implicaciones en su enseñanza. Didáctica de las Ciencias Experimentales y Sociales, 21, 91-117. https://ojs.uv.es/index.php/dces/article/view/2428

Sousa, E., Quintino, V., Palhas, J., Rodrigues, A.M. \& Teixeira, J. (2016). Can environmental education actions change public attitudes? An example using the pond habitat and associated biodiversity. PLOS ONE, 11, e0154440. DOI:http://dx.doi.org/10.1371/journal.pone.0154440

Tacca-Huamán, D. R. (2011). La enseñanza de las ciencias naturales en la educación básica. Investigación Educativa, 14, 139-152. Recuperado de: https://educrea.cl/wp-content/uploads/2016/07/DOC1-ensenanza-de-lasciencias.pdf

Tribó, G. (2008). El nuevo perfil profesional de los profesores de secundaria. Educación $X X 1,11,183-209$. DOI: http://dx.doi.org/10.5944/educxx1.11.0.314

Van Weelie, D. \& Wals, A. (2002). Making biodiversity meaningful through environmental education. International Journal of Science Education, 24, 1143-1156. DOI: http://dx.doi.org/10.1080/09500690210134839

Vázquez-Alonso, A. \& Manassero-Mas, M.A. (2011). El descenso de las actitudes hacia la ciencia de chicos y chicas en la educación obligatoria. Ciencia \& Educação, 17, 249-268. http://dx.doi.org//10.1590/S1516-73132011000200001

Vega-Marcote, P. \& Álvarez-Suárez, P. (2009). Actitudes ambientales y conductas sostenibles. Implicaciones para la educación ambiental. Revista de Psicodidáctica, 14, 245-260. Recuperado de: http://www.ehu.eus/ojs/index.php/psicodidactica/article/view/727/603

Yli-Panula, E., Jeronen, E., Lemmetty, P. \& Pauna, A. (2018). Teaching methods in biology promoting biodiversity education. Sustainability, 10, 3812. DOI: http://dx.doi.org/10.3390/su10103812 